\title{
Differentiation of mouse embryonic stem cells to hepatocyte-like cells by co-culture with human liver nonparenchymal cell lines
}

\author{
Alejandro Soto-Gutiérrez ${ }^{1,5}$, Nalu Navarro-Álvarez ${ }^{1,5}$, Debiao Zhao $^{2}$, Jorge D Rivas-Carrillo ${ }^{1}$, Jane Lebkowski ${ }^{3}$, \\ Noriaki Tanaka ${ }^{1}$, Ira J Fox ${ }^{4} \&$ Naoya Kobayashi ${ }^{1}$ \\ ${ }^{1}$ Department of Surgery, Okayama University Graduate School of Medicine and Dentistry, 2-5-1 Shikata-cho, Okayama 700-8558, Japan. ${ }^{2}$ Roslin Institute, Roslin, \\ Midlothian, EH25 9PS, U.K. ${ }^{3}$ Geron Corporation, 230 Constitution Drive, Menlo Park, California 94025, USA. ${ }^{4}$ Department of Surgery, University of Nebraska Medical \\ Center, Omaha, Nebraska 68198-3285, USA. ${ }^{5}$ These authors contributed equally to this work. Correspondence should be addressed to I.J.F. (ijfox@unmc.edu) or \\ N.K. (immortal@md.okayama-u.ac.jp).
}

Published online 8 March 2007; doi:10.1038/nprot.2007.18

This protocol describes a co-culture system for the in vitro differentiation of mouse embryonic stem cells into hepatocyte-like cells. Differentiation involves four steps: (i) formation of embryoid bodies (EB), (ii) induction of definitive endoderm from 2-d-old EBs, (iii) induction of hepatic progenitor cells and (iv) maturation into hepatocyte-like cells. Differentiation is completed by $16 \mathrm{~d}$ of culture. EBs are formed, and cells can be induced to differentiate into definitive endoderm by culture in Activin A and fibroblast growth factor 2 (FGF-2). Hepatic differentiation and maturation of cells is accomplished by withdrawal of Activin A and FGF-2 and by exposure to liver nonparenchymal cell-derived growth factors, a deleted variant of hepatocyte growth factor (dHGF) and dexamethasone. Approximately $70 \%$ of differentiated embryonic stem (ES) cells express albumin and can be recovered by albumin promoter-based cell sorting. The sorted cells produce albumin in culture and metabolize ammonia, lidocaine and diazepam at approximately two-thirds the rate of primary mouse hepatocytes.

\section{INTRODUCTION}

Protocols have been developed to differentiate and enrich various cell types from embryonic stem (ES) cells. Previous studies indicate that ES cells can be differentiated into hepatocyte-like cells ${ }^{1-6}$. ES cell-derived hepatocytes would be useful as a source of material for cell transplantation, bioartificial liver (BAL) support devices, studies on hepatocyte biology and hepatitis viruses and for drug testing. Methods for hepatic differentiation of ES cells can be divided into those involving spontaneous differentiation and those involving directed differentiation. Spontaneous differentiation of ES cells can be accomplished in serum-containing medium through the formation of embryoid bodies (EB)-containing cells representing ectoderm, mesoderm and endoderm lineages. Subsequent inoculation of the EB-derived cells on adherent matrix substrates allows spontaneous differentiation, producing occasional hepatocyte-like cells ${ }^{3,6}$. In vivo differentiation of mouse ES cells has also been accomplished by transplantation into the livers of carbon tetrachloride-treated mice. ES cell-derived hepatocyte precursors have been recovered from the intrahepatic teratomas that form following injury to the liver and ES cell engraftment ${ }^{1}$. These protocols have contributed to our understanding of factors affecting hepatic differentiation by mouse ES cells.

While a number of in vitro differentiation protocols (using retinoic acid (RA), hepatocyte growth factor (HGF), fibroblast growth factor-1 (FGF-1), FGF-4, oncostatin $M$ and various matrix substrates $^{2}$ ) have successfully generated hepatocyte-like cells, none has been shown to produce cells with a mature phenotype. The recent report of a technique for efficient generation of definitive endoderm from ES cells by culture in lower concentrations of serum and Activin $A^{7-9}$ has been an important advance for subsequent production of hepatocyte-like cells. We have found that induction of definitive endoderm from ES cells was a necessary step for subsequent hepatic differentiation in vitro. Hepatic differentiation of stem cells and maturation of hepatic progenitors occurs in vitro after co-culture with damaged slices of liver or culture in medium conditioned by nonparenchymal liver cells ${ }^{10-13}$. Reproducibility, however, has been limited.

We devised a strategy for differentiation of ES cells into hepatocyte-like cells using a four-step differentiation protocol: (i) generation of EBs; (ii) induction of definitive endoderm from 2-d-old EBs by growth in Activin A and FGF-2; (iii) induction of hepatic progenitors by co-culture with human liver nonparenchymal cell lines (human liver endothelial (TMNK-1) ${ }^{14}$, stellate $(\mathrm{TWNT}-1)^{15}$ and cholangiocyte $(\mathrm{MMNK}-1)^{16}$ cell lines) and a deleted variant of HGF (dHGF); and (iv) maturation into hepaticlike cells by culture in DMSO and dexamethasone (Fig. 1). This in vitro differentiation system leads to a modest degree of hepatocyte-specific gene expression after $7 \mathrm{~d}$ of culture and a significantly increased degree of hepatocyte-specific gene expression by $14 \mathrm{~d}$ of culture. After completion of the differentiation program, $68.7 \% \pm 4.8 \%$ (standard deviation) of cells express albumin. At day 7 of the differentiation program, $48.4 \pm 2.4 \%$ of the cells co-express alpha-fetoprotein (AFP), while on day 14 of the program only $21.3 \pm 3.7 \%$ of the cells co-express AFP. Differentiated ES cells secrete albumin; metabolize ammonia, lidocaine and diazepam; contain glycogen rosettes and express no pancreatic exocrine proteins. Mice with liver failure treated with an implantable liver assist device containing the ES cell-derived hepatocytes survived better and had improved liver function earlier than mice treated with the implantable assist device containing control cells ${ }^{17}$. The present hepatic differentiation strategy appears to require further optimization to eliminate AFP-positive cells and to produce a more homogeneous population of hepatocyte-like cells. It is possible that our protocol could be modified to obtain other cellular phenotypes by changing the culture conditions to include other types of cells. 
a

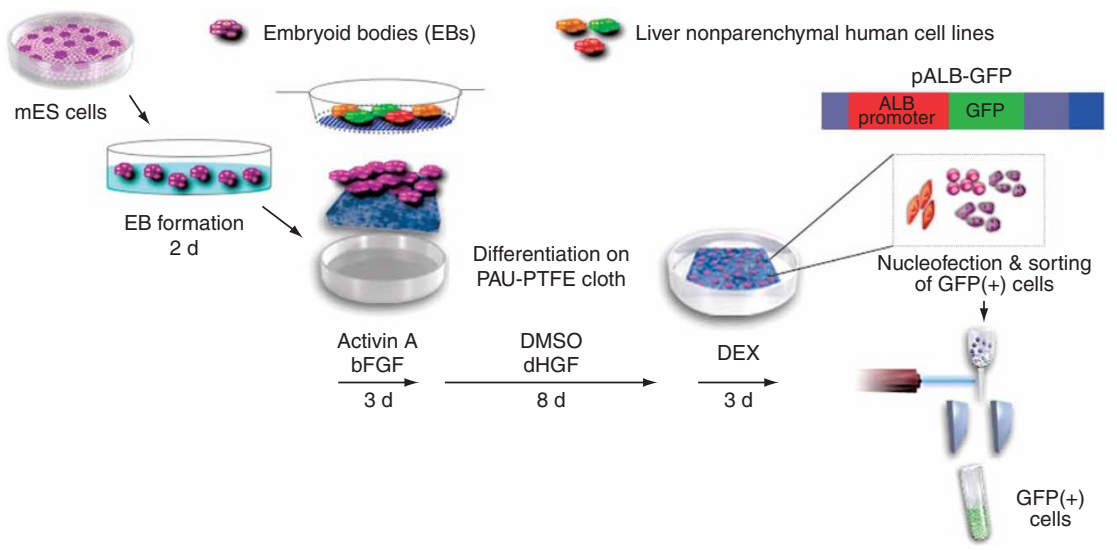

b

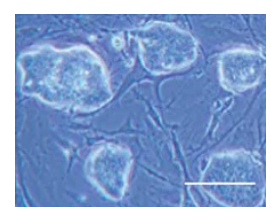

C
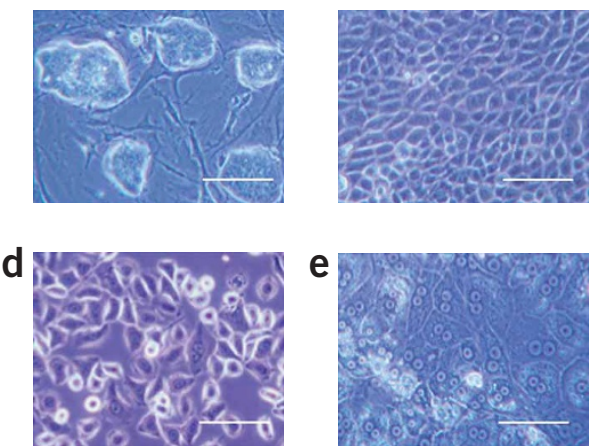

Figure 1 | Schematic representation of the strategy for differentiation of mouse embryonic stem cells to hepatocytes and their use for BAL therapy.

(a) Mouse embryonic stem cells ( $\mathrm{mES}$ cells) were cultured in suspension for $2 \mathrm{~d}$ to form embryoid bodies (EBs). The resulting EBs were transferred onto unwoven polytetrafluoroethylene (PTFE) cloth and treated with FGF-2 $(100 \mathrm{ng} / \mathrm{ml})$ and Activin-A $(100 \mathrm{ng} / \mathrm{ml})$ for $3 \mathrm{~d}$ and then co-cultured with mitomycin-C-treated human nonparenchymal liver cell lines on Matrigel-layered transwell membranes with the deleted variant of hepatocyte growth factor (dHGF; $100 \mathrm{ng} / \mathrm{ml})$ ) and $1 \%$ dimethylsulfoxide (DMSO) for $8 \mathrm{~d}$. The final stage of differentiation involves treatment with dexamethasone (DEX; $10^{-7} \mathrm{M}$ ) for $3 \mathrm{~d}$. At this stage, the cells are transduced with a plasmid containing GFP cDNA under the control of the albumin promoter (pALB-GFP) for selection of cells differentiated toward hepatocytes. (b-e) Phase-contrast photographs of undifferentiated mES cells and differentiating cells are shown. (b) Undifferentiated mES cells. (c) Definitive endoderm committed-cells. (d) Terminally differentiated cells (pALB-GFP-sorted cells). (e) Isolated primary mouse hepatocytes (original magnification: $100 \times$; bar: $50 \mu \mathrm{m}$ ).

\section{MATERIALS}

\section{REAGENTS}

$\triangle$ CRITICAL All reagents and materials should be sterile.

- Mouse ES cell line, strain Balb/c × 129sv (Dainippon Pharmaceutical Co., cat. no. CMTI-1)

- Primary mouse embryo fibroblasts (Dainippon Pharmaceutical Co., cat. no. PMEF-HL)

- Ultrapure water with $0.1 \%$ gelatin (Specialty Media, cat. no. ES-006B)

- Complete ES cell medium DMEM (Specialty Media, cat. no. ES-101-B)

- Leukemia inhibitory factor (LIF) (Chemicon, cat. no. ESG1106)

- Penicillin/streptomycin solution (Sigma, cat. no. P0781)

- Mitomycin C (Biomol, cat. no. GR-311)

- DMEM-F12 (Gibco, cat. no. 11320-033)

- Activin A (R\&D, cat. no. 338-AC)

- FGF-2 (Preprotech, cat. no. 100-18B)

- Deleted variant of hepatocyte growth factor (Daiichi Pharmaceutical Co.)

- Recombinant human hepatocyte growth factor (HGF) (Sigma, cat. no.

H9661) (optional)

- DMSO (Sigma, cat. no. 154938)

- Dexamethasone (Sigma, cat. no. D-8893)

- Reversibly immortalized human liver endothelial cell line (TMNK-1) ${ }^{14}$, human stellate cell line (TWNT-1) ${ }^{15}$ and human cholangiocyte cell line (MMNK-1) ${ }^{16}$. (TMNK-1, TWNT-1 and MMNT-1 may be obtained from the authors on agreement with a Material Transfer Agreement, available in Supplementary Material Transfer Agreement)

- Matrigel (Becton Dickinson, Bioscience, cat. no. 354234) (optional)

- Trypsin-EDTA (Sigma, cat. no. T4049)

- Ammonium sulfate (Nicalai Tesque, cat. no. 02619-15)

- Lidocaine (Fujisawa, cat. no. 3940)

- Diazepam (Takeda, cat. no. 5887)

- Trizol (Invitrogen, cat. no. 15596-026)

- ELISA kit: mouse albumin (Shibayagi, cat. no. AKRAL-121)

-Mounting medium with 4,6-diamidino-2-phenylindole (DAPI) (Vector, cat. no. VEC H-1200)

- Paraformaldehyde, formalin and ethanol

- Protease fluorescence detection kit (e.g., Sigma, cat. no. PF0100)

- Human protein cytokine array kit (Ray Biotech, cat. no. RAY H108010C)

- Plasmid pALB-GFP (see REAGENT SETUP)

\section{EQUIPMENT}

- Six-well plates (Falcon, cat. no. 353046)

$\cdot 0.4 \mu \mathrm{m}$ filter transwell inserts (Falcon, cat. no. 353039)
- Ultra-low attachment plates (Corning, cat. no. 3471)

- Spectrophotometer multiscan ELISA reader (Electron)

- Unwoven polytetrafluoroethylene (PTFE) cloth coated with poly-

aminourethane (PAU) (Kuraray Medical Co.) (see EQUIPMENT SETUP)

- Inverted confocal laser-scanning microscope (LSM510; Carl Zeiss)

- ImageJ software (US National Institutes of Health)

- Fluorocytometer (650-10S, Hitachi)

\section{REAGENT SETUP}

$\triangle$ CRITICAL All reagents should be freshly prepared.

Plasmid pALB-GFP This vector contains the gene encoding enhanced GFP driven from the human albumin (ALB) promoter. The DNA sequence and map for pALB-GFP are shown in Supplementary Figures 1 and 2. The pALB-GFP plasmid is available from D. Zhao (Centre for Regenerative Medicine, GU507, Chancellor's Building, 49 Little France Crescent, Edinburgh, EH16 4SB UK; e-mail: dzhao@staffmail.ed.ac.uk).

Mouse ES cell culture medium Complete ES culture medium (DMEM) (Specialty Media) containing 15\% FBS, $1 \%$ nonessential amino acid (1:100 dilution of the $100 \times$ stock), $1 \%$ nucleosides (1:100 dilution of the $100 \times$ stock), 2-mercaptoethanol $(110 \mu \mathrm{M}), 1 \%$ penicillin/streptomycin (100 units/ml penicillin, $0.1 \mathrm{mg} / \mathrm{ml}$ streptomycin), $1 \%$ glutamic acid (1:100 dilution of the $100 \times$ stock) and $500 \mathrm{U} / \mathrm{ml}$ leukemia inhibitory factor.

Mouse feeder cell medium DMEM (Sigma) containing 10\% FBS, 4,500 mg/l glucose, $2 \mathrm{mM}$ L-glutamine, $1 \%$ penicillin/streptomycin (100 units $/ \mathrm{ml}$ penicillin, $0.1 \mathrm{mg} / \mathrm{ml}$ streptomycin).

Human liver cell line medium containing mitomycin C DMEM (Sigma) containing 10\% FBS, 4,500 mg/l glucose, $2 \mathrm{mM}$ L-glutamine, $1 \%$ penicillin/ streptomycin (100 units/ml penicillin, $0.1 \mathrm{mg} / \mathrm{ml}$ streptomycin) and mitomycin $\mathrm{C}(10 \mu \mathrm{g} / \mathrm{ml})$.

Embryoid body formation medium DMEM-F12 (Gibco) containing 15\% FBS, $1 \%$ nonessential amino acids (1:100 dilution of the $100 \times$ stock), $1 \%$ nucleosides (1:100 dilution of the $100 \times$ stock), $1 \%$ penicillin/streptomycin (100 units $/ \mathrm{ml}$ penicillin, $0.1 \mathrm{mg} / \mathrm{ml}$ streptomycin) and $1 \%$ glutamic acid (1:100 dilution of the $100 \times$ stock).

Differentiation medium I DMEM-F12 (Gibco) containing 1\% FBS, 1\% nonessential amino acids (1:100 dilution of the $100 \times$ stock), $1 \%$ nucleosides ( $1: 100$ dilution of the $100 \times$ stock), $1 \%$ penicillin/streptomycin (100 units $/ \mathrm{ml}$ penicillin, $0.1 \mathrm{mg} / \mathrm{ml}$ streptomycin), $1 \%$ glutamic acid (1:100 dilution of the $100 \times$ stock), 3\% BSA, $100 \mathrm{ng} / \mathrm{ml}$ FGF-2 and $100 \mathrm{ng} / \mathrm{ml}$ Activin-A.

Differentiation medium II DMEM-F12 (Gibco) containing 10-15\% FBS, 1\% nonessential amino acid (1:100 dilution of the $100 \times$ stock), $1 \%$ nucleosides 
(1:100 dilution of the $100 \times$ stock), $1 \%$ penicillin/streptomycin $(100$ units $/ \mathrm{ml}$ penicillin, $0.1 \mathrm{mg} / \mathrm{ml}$ streptomycin), $1 \%$ glutamic acid (1:100 dilution of the $100 \times$ stock), $100 \mathrm{ng} / \mathrm{ml} \mathrm{dHGF}$ or $10-50 \mathrm{ng} / \mathrm{ml} \mathrm{HGF}$ and $1 \%$ DMSO.

Differentiation medium III DMEM-F12 (Gibco) containing 10-15\% FBS, $1 \%$ nonessential amino acid (1:100 dilution of the $100 \times$ stock), $1 \%$ nucleosides ( $1: 100$ dilution of the $100 \times$ stock), $1 \%$ penicillin/streptomycin $(100$ units $/ \mathrm{ml}$ penicillin, $0.1 \mathrm{mg} / \mathrm{ml}$ streptomycin), $1 \%$ glutamic acid (1:100 dilution of the $100 \times$ stock), $100 \mathrm{ng} / \mathrm{ml} \mathrm{dHGF}$ and $10^{-7} \mathrm{M}$ dexamethasone.

Cell lines The TMNK- 1 cell line was generated from commercially available normal human liver endothelial cells (Dinippon Sumitomo Parma, cat. no. CS-ABI-566) by transduction with recombinant retrovirus vectors SSR\#69, which transfers expression of the simian virus 40 large T antigen (SV40T), and SSR\#197, which transfers human telomerase reverse transcriptase (hTERT) expression. The TWNT-1 cell line was generated from human LI 90 cells, which were derived from a mesenchymal liver tumor removed from a 55 -year-old Japanese woman. The cells were transduced with the SSR\#197 recombinant retroviral vector, which transfers human telomerase reverse transcriptase (hTERT) expression. The MMNK-1 cell line was generated from OUMS-21 cells, which were derived from human fetal liver by transduction with the SSR\#197 recombinant retrovirus vector (hTERT). TMNK-1, TWNT-1, and MMNK-1 cell lines were deposited as FERM BP-8017 on April 16, 2002, FERM BP- 7843 on January 8, 2002 and FERM BP-8126 on August 1, 2002, respectively, with the International Patent Organism Depository, National Institute of Advanced Industrial Science and Technology, Japan.

EQUIPMENT SETUP

Matrigel-coated plates Slowly thaw Matrigel aliquots at $4{ }^{\circ} \mathrm{C}$ for at least $2 \mathrm{~h}$ to avoid the formation of a gel. Dilute Matrigel in DMEM-F12 (serum-free) for a final dilution of 1:30. Add $1 \mathrm{ml}$ of Matrigel solution to coat each well of a six-well plate. Incubate the plates for $1-2 \mathrm{~h}$ at room temperature (RT), or at least overnight at $4{ }^{\circ} \mathrm{C}$. Plates with Matrigel solution can be stored at $4{ }^{\circ} \mathrm{C}$ for 1 week. Remove Matrigel solution immediately before use.

Unwoven polytetrafluoroethylene (PTFE) cloth coated with polyaminourethane (PAU) Information concerning the preparation and properties of the unwoven PTFE cloth coated with PAU is available from Japanese Patent no. 11-239612. The material is available from Kuraray Medical Co., Japan.

\section{PROCEDURE}

\section{Mouse ES cell culture}

1) Prepare mouse feeder cells $1 \mathrm{~d}$ before passage or thawing of ES cells. Inactivate feeder cells before use with ES cells by incubating for $3 \mathrm{~h}$ with medium containing mitomycin $\mathrm{C}(10 \mu \mathrm{g} / \mathrm{ml})$ and washing with PBS two or three times.

$\triangle$ CRITICAL STEP Use feeder cells within 1 week of preparation.

2| Plate mouse ES cells on feeder cells at $4 \times 10^{4} \mathrm{ES}$ cells $/ \mathrm{cm}^{2}$ of culture area. Then exchange culture medium every day, adding LIF (500 U/ml), and passage the ES cells every $3 \mathrm{~d}$.

3| For subculture, after $72 \mathrm{~h}$ of culture, remove medium and wash the cell layer with PBS.

4| Add $0.5 \mathrm{ml}$ trypsin/EDTA solution, wait $30 \mathrm{~s}$ at RT and remove the trypsin/EDTA solution. By this operation, the feeder cells are removed and only the ES cells remain in the flask.

5| Two minutes later, observe the ES cells detach in the microscope and then add $5 \mathrm{ml}$ of fresh ES cell culture medium.

6| Resuspend the cell population into a single cell suspension and centrifuge at $100 \mathrm{~g}$ for $1 \mathrm{~min}$. Resuspend the cells in medium and plate cells on inactivated feeder cells (adjusting cell number as required)

$\triangle$ CRITICAL STEP Reproducible ES cell differentiation depends strongly on the quality of ES cells used. For that reason, exchange the medium for embryonic stem cells every day, because the metabolic activity of mouse ES cells is high. Differentiation capacity may decrease if passage is performed less often than every $3 \mathrm{~d}$.

\section{Generation of embryoid bodies from mouse ES cell cultures}

7| Trypsinize ES cells as described in Steps 3-6 above, except resuspend cells in $2 \mathrm{~mL}$ of EB formation medium at the end. Transfer the single-cell solution to one well of an ultra-low attachment plate. Add $2 \mathrm{ml}$ of EB formation medium to each well to give a total volume of $4 \mathrm{ml}$ per well. Depending on the cell density of the ES cells, the split ratio for this procedure can vary. In our hands, a cell suspension concentration of $1.0 \times 10^{5} \mathrm{cells} / \mathrm{ml}$ medium is suitable for hepatic differentiation.

8| Optionally, change the EB formation medium to fresh medium after $24 \mathrm{~h}$. To do this, transfer the EBs into a 15-ml tube and centrifuge at $100 \mathrm{~g}$ for $3 \mathrm{~min}$. Aspirate the supernatant, replace with fresh EB formation medium ( $4 \mathrm{ml} / \mathrm{well})$ and transfer to six-well ultra-low attachment plates for embryoid body formation. After $48 \mathrm{~h}$ of culture in suspension, check if ES cells have formed floating aggregates known as embryoid bodies (EBs).

$\triangle$ CRITICAL STEP A low number of cells per well leads to small-size EBs with insufficient differentiation capacity. An excessive number of ES cells per well results in large EBs with apoptotic cells. The number of mouse ES cells should be adjusted per well. EBs are formed before treatment with Activin A to reduce the cytotoxicity associated with treatment with low serum and Activin A. This cytotoxicity occurs even in the presence of high concentrations of FGF-2 (100 ng/ml). ES-derived hepatocytes also have better hepatocyte-like function when EBs are formed first.

9| Transfer the EBs into a $15-\mathrm{ml}$ tube and let aggregates settle for $5 \mathrm{~min}$, or centrifuge at $100 \mathrm{~g}$ for $3 \mathrm{~min}$. Aspirate the supernatant, replace with fresh differentiation medium $(2 \mathrm{ml} /$ well) and transfer to six-well plates for further differentiation.

\section{Induction of definitive endoderm}

10| Prepare unwoven polytetrafluoroethylene (PTFE) cloth coated with polyaminourethane (PAU) and place into dishes, or, optionally, use Matrigel-coated dishes (see REAGENT SETUP). We have obtained better differentiation and specificity 
when using PAU-coated PTFE, but Matrigel can be also used because it is a major component of the extracellular matrix. We believe that the human liver nonparenchymal cell lines secrete necessary matrix components into the medium for hepatic differentiation.

11 After transferring EBs to individual wells of six-well plates, add $2 \mathrm{ml}$ of differentiation medium I for $3 \mathrm{~d}$. If clusters of cells are not attached, change medium to fresh medium after the second day (optional). Collect the medium into a 15-ml tube and let aggregates settle for $5 \mathrm{~min}$. Aspirate the supernatant, replace with fresh differentiation medium and plate again in the same well. $\triangle$ CRITICAL STEP FBS is needed for cell attachment so you can culture EBs for $8 \mathrm{~h}$ in $2 \mathrm{ml}$ of differentiation medium I supplemented with $10 \%$ FBS on Matrigel before the next step. Remove FBS after cell attachment.

$\triangle$ CRITICAL STEP Individual EBs should have sufficient space to allow cells to proliferate and to migrate for several days after plating. If the cell density is too high, it might lead to insufficient differentiation owing to metabolic starvation, cell necrosis and/or apoptosis or might lead to formation of tissue-like aggregates at the end of the differentiation protocol that will be very difficult to dissociate into a single-cell suspension.

\section{Induction of hepatic progenitor cells (expressing both albumin and AFP) and maturation into hepatocyte-like cells by co-culture with human liver nonparenchymal cell lines}

12| Prepare human liver nonparenchymal cell lines ${ }^{14-16}$, which can be maintained in T75 tissue culture flasks in $10 \mathrm{~mL}$ of DMEM and $10 \%$ FBS. Passage confluent cultures every week, and change the medium every $3 \mathrm{~d}$.

13| Before co-culture, treat confluent cell lines with human liver cell line medium containing mitomycin $\mathrm{C}$ for $2 \mathrm{~h}$. Then wash cells two or three times with PBS.

14| To harvest cells, remove culture medium, wash with PBS and treat adherent cell lines with $1.5 \mathrm{~mL}$ of trypsin/EDTA solution to detach them from the plastic surface. Incubate the flask for 3-5 $\mathrm{min}$ at $37^{\circ} \mathrm{C}$. Confirm by visual inspection that the majority of cells are in suspension.

15 Add $10 \mathrm{ml}$ of DMEM 10\% FBS and resuspend the cells by gentle pipetting. Transfer the cell suspension to a 15-ml plastic tube. Centrifuge immediately at $4{ }^{\circ} \mathrm{C}(200 \mathrm{~g}$ for $5 \mathrm{~min})$.

16 Discard the supernatant and resuspend the cell pellet in $10 \mathrm{ml}$ fresh culture medium. Add $1 \mathrm{ml}$ of the cell suspension into a new 775 flask containing $9 \mathrm{ml}$ of fresh culture medium for further culture. The number of cells from each cell line that grows in a T75 culture flask varies depending on confluence. For that reason, count cells for transwell co-culture.

17| Distribute $5 \times 10^{5}$ mitomycin C-treated, reversibly immortalized human cholangiocytes (MMNK-1 cells), $5 \times 10^{5}$ liver endothelial cells (TMNK-1 cells) and $5 \times 10^{5}$ hepatic stellate cells (TWNT-1 cells), suspended in $1.5 \mathrm{ml}$ of differentiation medium II, in one $0.4-\mu \mathrm{m}$ transwell. Using sterile forceps, place one transwell into each well containing differentiating EBs. Make sure no air bubbles are trapped under the transwell membrane.

$\triangle$ CRITICAL STEP Do not use human liver cell lines cells that are treated with mitomycin C more than 1 week ahead of time. Use sterile technique.

18| Add 2-3 ml of differentiation medium II supplemented with $10 \%$ FBS, and change the medium every day. Replace transwells containing new mitomycin C-treated human liver nonparenchymal cell lines every second or third day.

19| After $8 \mathrm{~d}$ of culture in differentiation medium II, remove medium and replace with differentiation medium III supplemented with dexamethasone (DEX). The last $3 \mathrm{~d}$ of differentiation require use of differentiation medium III supplemented with DEX.

20| After differentiation, trypsinize cells, suspend them as single cells and transfect them using Nucleofector, according to the manufacturer's specifications, with a plasmid vector containing GFP cDNA under the control of an albumin promoter (pALB-GFP). Determine GFP expression using a DakoCytomation MoFlo or BD FACSaria cell sorter, and recover GFP-positive cells for further studies by MoFlo. At any stage of differentiation, cells can be trypsinized and analyzed by immunohistochemistry (option C), RT-PCR (option A) or ELISA analysis (option B). Levels of cytokines in the medium can also be determined (option D). Proteases can be detected in the culture supernatant of hepatocyte-like cells derived from mouse ES cells by option $E$, using a protease fluorescence detection kit (Sigma) to detect serine proteases, cysteine proteases, metalloproteases and aspartic proteases in the culture medium.

$\triangle$ CRITICAL STEP Results may vary if optional reagents are used, such as cell culture Matrigel or use of HGF rather than dHGF (see Supplementary Figs. 10 and 11 from ref. 17).

(A) Gene expression analyses of hepatocyte-like cells differentiated from mouse ES cells.

(i) Extract total RNA from a minimum of $1 \times 10^{6}$ cells at any stage of the differentiation protocol, using Trizol according to the manufacturer's protocol.

(ii) Perform reverse transcription (RT)-PCR, at $22{ }^{\circ} \mathrm{C}$ for 10 min and then at $42{ }^{\circ} \mathrm{C}$ for 20 min using $1.0 \mu \mathrm{g}$ of RNA per reaction. Conditions can vary depending on the thermal cycler that is used. Amplify the cDNA through 25-40 thermal cycles. 
TABLE 1 | Selected RT-PCR primer sequences and GenBank accession numbers.

\begin{tabular}{|c|c|c|}
\hline Gene & Primer sequence (forward/reverse) & GenBank accession \\
\hline \multirow[t]{2}{*}{ Albumin } & 5'- GACAAGGAAAGCTGCCTGAC-3' & AJ011413 \\
\hline & 5'- TTCTGCAAAGTCAGCATTGG-3' & \\
\hline CK 18 & 5'-CGATACAAGGCACAGATGGA-3' & XM356490 \\
\hline \multirow[t]{2}{*}{ FOXA2 } & 5'-TATTGGCTGCAGCTAAGCGG-3' & U04197 \\
\hline & 5'-GACTCGGACTCAGGTGAGGT-3' & \\
\hline S0X17 & 5'-TTTGTGTATAAGCCCGAGATGG-3' & NM011441 \\
\hline \multirow[t]{2}{*}{ CYP 7A1 } & 5'-AGGACTTCACTCTACACC- $3^{\prime}$ & AK050260 \\
\hline & 5'-GCAGTCGTTACATCATCC-3' & \\
\hline \multirow[t]{2}{*}{ AFP } & 5'-CACTGCTGCAACTCTTCGTA-3' & V00743 \\
\hline & 5'-CTTTGGACCCTCTTCTGTGA-3' & \\
\hline \multirow[t]{2}{*}{ NKx2.5 } & $5^{\prime}$-CAGTGGAGCTGGACAAAGCC-3' & NM008700 \\
\hline & 5'-TAGCGACGGTTCTGGAACCA-3' & \\
\hline \multirow[t]{2}{*}{ Cdh3 } & 5'- AAACTTGGGGACAGCAACATCAG-3' & \\
\hline & 5'-TCTTTTGGTTTGCAGAGACAGGG-3' & NM001037809 \\
\hline \multirow[t]{2}{*}{ Brachyury } & 5'-AACTTTCCTCCATGTGCTGAGAC-3' & NM009309 \\
\hline & 5'-TGACTTCCCAACACAAAAAGCT-3' & \\
\hline \multirow[t]{2}{*}{ FOXA1 } & $5^{\prime}$ - GGCCTGGGCTCTATGAACTC-3' & NM008259 \\
\hline & $5^{\prime}$-GCCGTTCATGGAGGTGAC-3' & \\
\hline \multirow[t]{2}{*}{ GAPDH } & 5'-TGAAGGTCGGTGTGAACGGATTTGGC-3' & NM001001303 \\
\hline & 5'-TGTTGGGGGCCGAGTTGGGATA-3' & \\
\hline
\end{tabular}

Standard conditions are denaturation at $95{ }^{\circ} \mathrm{C}$ for $40 \mathrm{~s}$, annealing at $55-68{ }^{\circ} \mathrm{C}$ for $40 \mathrm{~s}$ and extension at $72{ }^{\circ} \mathrm{C}$ for $40 \mathrm{~s}$. The conditions depend on the primers and thermal cycler used. Cool samples to $4{ }^{\circ} \mathrm{C}$ and store at $-20{ }^{\circ} \mathrm{C}$ (Table 1).

PAUSE POINT RT-PCR products can be stored at $-20{ }^{\circ} \mathrm{C}$ for as long as 24 weeks.

(iii) Resolve PCR products on $1 \%$ agarose gels and visualize by ethidium bromide staining $(0.4 \mu \mathrm{g} / \mathrm{ml})$. Illuminate the gel by UV light and obtain a digital image.

! CAUTION Ethidium bromide is carcinogenic. Wear gloves and dispose of all contaminated pipette tips, agarose gels and buffers separately.

(B) Evaluation of metabolic capacity and albumin secretion.

(i) Seed $5 \times 10^{5}$ hepatocyte-like cells derived from mouse ES cells into wells of a 12-well tissue culture plate. Individual wells can be coated with either a PAU-treated PTFE cloth or Matrigel,

(ii) Twenty-four hours later, add $2 \mathrm{ml}$ of differentiation culture medium III supplemented with ammonium sulfate $(0.56 \mathrm{mM})$, lidocaine $(1 \mathrm{mg} / \mathrm{ml})$ or diazepam $(1 \mu \mathrm{g} / \mathrm{ml})$ to individual wells.

(iii) Twenty-four hours later, measure the amounts of each chemical in the culture medium. The ammonia concentration can be determined using a Fuji Dri-Chem slide, and concentrations of lidocaine and diazepam can be measured by a fluorescence polarization immunoassay and high-performance liquid chromatography (SRL).

(iv) To measure $24 \mathrm{~h}$ albumin secretion into the culture medium, use a mouse albumin ELISA kit (Shibayagi). The reaction is stopped by adding acid to give a colorimetric endpoint that is read spectrophotometrically.

TABLE 2 | Selected primary antibodies to characterize progenitor and mature pancreatic and hepatic cell types.

\begin{tabular}{lll}
\hline Primary antibody & Dilution & Supplier \\
\hline FITC-conjugated rabbit antibody to mouse albumin & $1: 100$ & Cedarlane laboratories \\
Rabbit antibody to human AFP & $1: 100$ & Santa Cruz \\
Rabbit antibody to human CYP7A1 & $1: 100$ & Santa Cruz \\
Rabbit antibody to mouse HNF4 & $1: 100$ & Santa Cruz \\
Rabbit antibody to mouse OCT3/4 & $1: 100$ & Santa Cruz \\
Goat antibody to human amylase & $1: 100$ & Santa Cruz \\
Goat antibody to mouse secretin & $1: 100$ & Santa Cruz \\
Goat antibody to human gastrin & $1: 100$ & Santa Cruz \\
\hline
\end{tabular}


TABLE 3 | Selected fluorescence-labeled secondary antibodies.

\begin{tabular}{lll}
\hline Secondary antibody & Dilution & Supplier \\
\hline Alexa 488-conjugated goat antibody to rabbit IgG & $1: 100$ & Molecular Probes \\
Alexa 594-conjugated donkey antibody to goat IgG & $1: 100$ & Molecular Probes \\
Alexa 594-conjugated goat antibody to rabbit IgG & $1: 100$ & Molecular Probes \\
\hline
\end{tabular}

(v) For determination of total protein, extract proteins with acid ethanol by overnight incubation at $4{ }^{\circ} \mathrm{C}$. After cell sonication, store at $-20{ }^{\circ} \mathrm{C}$ until determination of total protein content. Determine total protein content by Bradford assay according to manufacturer's specifications.

$\triangle$ CRITICAL STEP To avoid overestimation of albumin secretion, cells must be incubated in serum-free medium and should be thoroughly washed before assessing by ELISA.

\section{(C) Immunofluorescence analysis}

(i) Fix the cultured cells with 4\% paraformaldehyde in PBS followed by treatment with ethanol. Fix tissues with formalin and embed in paraffin under conventional conditions.

(ii) Incubate samples with primary antibodies at $37^{\circ} \mathrm{C}$ for $1 \mathrm{~h}$ (dilutions of selected antibodies are indicated in Table 2).

(iii) Rinse cover slips with PBS at RT for 5 min.

(iv) Apply second antibody (depending on the primary antibody; see Table 3), diluted in PBS containing $0.5 \%$ BSA, in a humidified chamber at $37^{\circ} \mathrm{C}$ for $45 \mathrm{~min}$.

(v) To label the cell nuclei, incubate specimens with mounting medium, containing DAPI or PI, at RT for 10 min.

(vi) Rinse cover slips twice with PBS at RT for 5 min.

(vii) Rinse cover slips quickly with distilled water at RT.

(viii) Embed cover slips in mounting medium and analyze immunolabeled cells with a conventional fluorescence and/or confocal laser-scanning microscope. DAPI-containing mounting medium can be used after washing the cover slips.

(D) Analysis of the human liver nonparenchymal cell conditioned medium using a human protein cytokine array

(i) Treat the membranes from the human protein cytokine array kit (Ray Biotech) with blocking buffer for 30 min.

(ii) Apply $2 \mathrm{ml}$ of serum-free medium from $1 \times 10^{6}$ cells (cultured overnight) from each of the human liver non parenchymal cell lines (TMNK-1 (endothelial cell line), MMNK-1 (cholangiocyte cell line) or TWNT-1 (stellate cell line)) at RT for $2 \mathrm{~h}$.

(iii) Wash membranes and incubate with $1 \mathrm{ml}$ of the primary biotin-conjugated antibody for $2 \mathrm{~h}$ at RT.

(iv) Incubate membranes with $2 \mathrm{ml}$ horseradish peroxidase-conjugated streptavidin for $30 \mathrm{~min}$ at RT.

(v) Expose membranes for development using an enhanced chemiluminescence-type solution. Expose membranes to film, and process by autoradiography as described by the manufacturer.

(vi) Calculate relative expression by ImageJ software (v. 1.33u).

(E) Protease detection in the culture supernatant of hepatocyte-like cells derived from mouse ES cells

(i) Collect $24 \mathrm{~h}$ culture medium from hepatocyte-like cells derived from mouse ES cells.

(ii) Incubate culture medium samples with buffer and the fluorescein isothiocyanate (FITC)-casein substrate for $12 \mathrm{~h}$ at $37^{\circ} \mathrm{C}$.

(iii) Acidify the mixture by adding trichloroacetic acid, and incubate at $37^{\circ} \mathrm{C}$ for $30 \mathrm{~min}$.

(iv) Centrifuge the mixture $(10 \mathrm{~min}$ at $10,000 \mathrm{~g})$ and remove the supernatant containing the FITC-labeled fragments.

(v) Measure with a fluorocytometer (e.g., Hitachi 650-10S) using excitation wavelengths of $485 \mathrm{~nm}$ and $535 \mathrm{~nm}$. Use serial dilutions of trypsin as a control, as described by the manufacturer.

\section{TIMING}

See Figure 1 for a timeline for production of hepatocyte-like cells (which takes $16 \mathrm{~d}$ ).

Gene expression analysis: $6 \mathrm{~h}$

Immunofluorescence: $4 \mathrm{~h}$

Mouse albumin ELISA: $7 \mathrm{~d}$

Protein cytokine array: $6 \mathrm{~h}$

Protease detection: $2 \mathrm{~d}$

\section{? TROUBLESHOOTING}

Troubleshooting advice can be found in Table 4.

TABLE 4 | Troubleshooting table.

\begin{tabular}{lll}
\hline Problem & Possible reason & Solution \\
\hline Loss of ES cell pluripotency & Feeder cells too old, LIF concentration & Start a new culture of feeder cells at an early passage \\
& too low or quality of LIF is insufficient & $\begin{array}{l}\text { and check the LIF concentration, or subculture mouse } \\
\text { ES cells at high density to avoid differentiation }\end{array}$
\end{tabular}


TABLE 4 | Troubleshooting table (continued).

\begin{tabular}{|c|c|c|}
\hline Problem & Possible reason & Solution \\
\hline $\begin{array}{l}\text { Inappropriate attachment of EBs on } \\
\text { PTFE-PAU or Matrigel }\end{array}$ & $\begin{array}{l}\text { Not sufficient culture time in the } \\
\text { presence of FBS }\end{array}$ & $\begin{array}{l}\text { Cell attachment depends of the presence of FBS. Culture } \\
\text { the cells at least } 8 \mathrm{~h} \text { in the presence of FBS, and then } \\
\text { change the medium for further differentiation }\end{array}$ \\
\hline $\begin{array}{l}\text { Nonspecific immunolabeling (specially for } \\
\text { albumin) }\end{array}$ & Nonspecific binding of antibodies & $\begin{array}{l}\text { Incubate the cells in medium without serum at least } 2 \mathrm{~h} \\
\text { before fixing. Omit primary antibody for negative } \\
\text { controls. Check cellular morphology and distribution } \\
\text { of immunofluorescence signals }\end{array}$ \\
\hline $\begin{array}{l}\text { Weak signal or no signal when performing } \\
\text { protein array from conditioned medium }\end{array}$ & $\begin{array}{l}\text { Too much time taken for detection, } \\
\text { sample too dilute or antibody cocktail } \\
\text { too dilute }\end{array}$ & $\begin{array}{l}\text { The whole detection process must be completed in } \\
\text { under } 30 \text { min. Increase sample volume to } 2 \mathrm{ml} \text {, check } \\
\text { that the cells are } 100 \% \text { confluent or, as an alternative, } \\
\text { slightly increase the amount of biotin antibody used } \\
\text { (diluted up to } 2 \mathrm{ml} \text { ) }\end{array}$ \\
\hline
\end{tabular}

\section{ANTICIPATED RESULTS}

\section{Gene expression, as determined by RT-PCR, and immunofluorescence analysis of 2-d-old EBs}

This four-stage protocol consists of (i) formation of embryoid bodies (EB), (ii) induction of definitive endoderm from $2 \mathrm{~d}$-old EBs, (iii) induction of hepatic progenitor cells and (iv) maturation into hepatocyte-like cells. Differentiation is completed by $16 \mathrm{~d}$ of culture (Fig. 1). Expression of endoderm- and hepatocyte-specific genes in 2-d-old embryoid bodies (D0 cells) using immunohistochemistry should not show expression of hepatocyte-specific markers, such as albumin and CYP7a1, but P0U5f1,
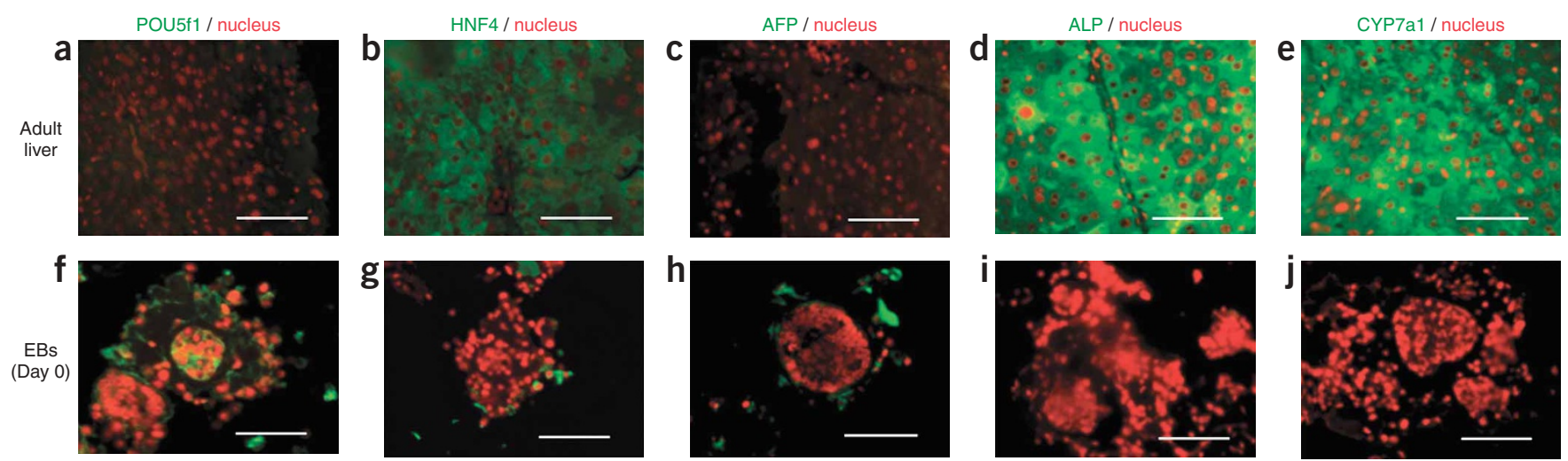

k
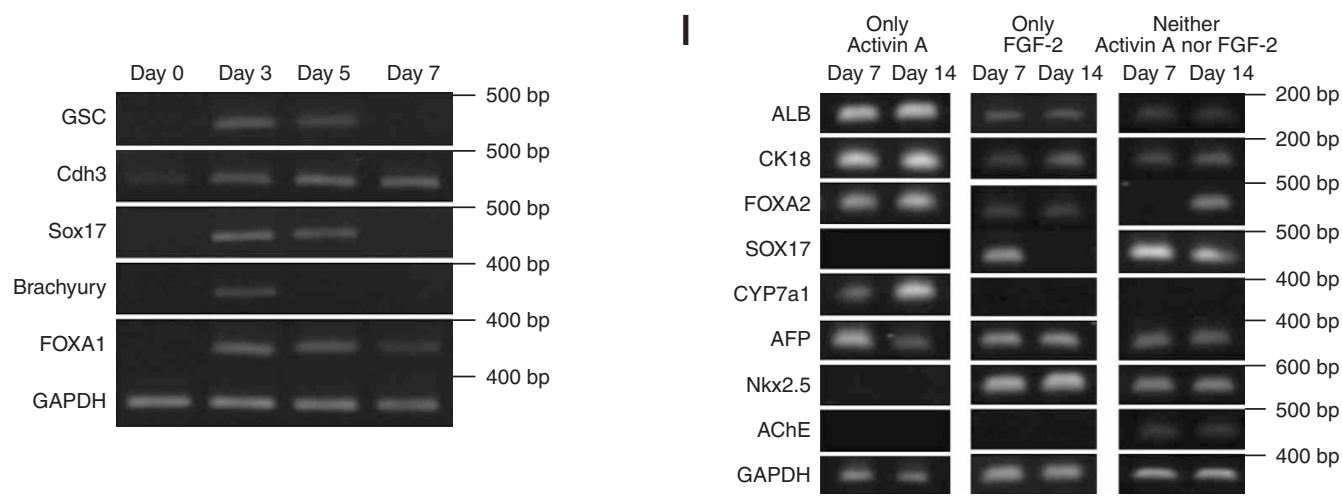

Figure 2 | Expression of primitive endoderm and hepatocyte-specific genes in 2-d-old embryoid bodies (D0 cells) using immunohistochemistry. Hepatocytespecific markers $(\mathbf{d}, \mathbf{e}, \mathbf{i}, \mathbf{j})$ albumin and CYP7a1 were not expressed, but POU5f1, a marker for undifferentiated ES cells (a,f), and the primitive endoderm markers HNF4 (b,g) and AFP $(\mathbf{c}, \mathbf{h})$ were expressed in EB cells. These findings are consistent with RT-PCR data from D0. (k) Characteristics of differentiating ES cell-derived hepatocytes by RT-PCR. RT-PCR analysis of gene expression in mES cells from day 0 to day 7 of differentiation shows induction of markers of definitive endoderm (GSC, Cdh3 and Sox17) and transient expression of the primitive streak gene brachyury, indicating induction of mesendoderm. (l) Gene expression profile during liver-specific ES cell differentiation showing the effect of culture in Activin A and fibroblast growth factor (FGF). EBs were cultured for $3 \mathrm{~d}$ with Activin-A only, FGF-2 only or with neither Activin-A nor FGF-2. Cells treated in this way were then cultured as outlined with dHGF, DMSO and DEX. RT-PCR gene expression data during liver-specific ES cell differentiation on days 7 and 14 of differentiation shows that Activin-A is essential for the initiation of hepatic differentiation. Bars, $50 \mu \mathrm{m}$. 
a

Human liver endothelial cell line (TMNK-1)
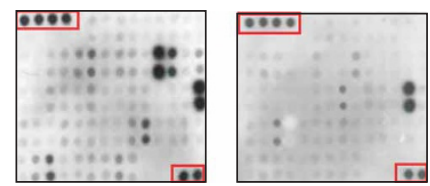

b

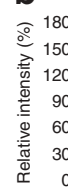

Human liver endothelial cell line conditioned medium
Human liver cholangiocyte cell line (MMNK-1)
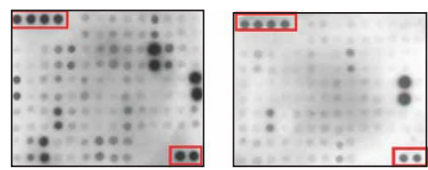

Human liver stellate cell line (TWNT-1)
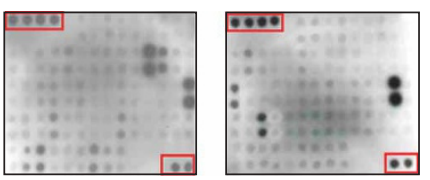

e

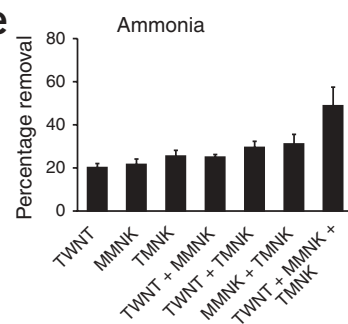

f

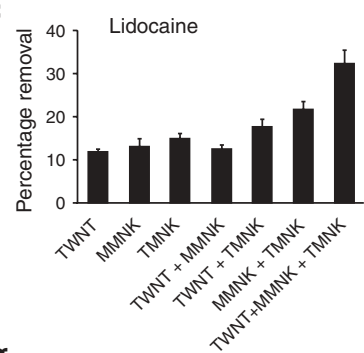

g

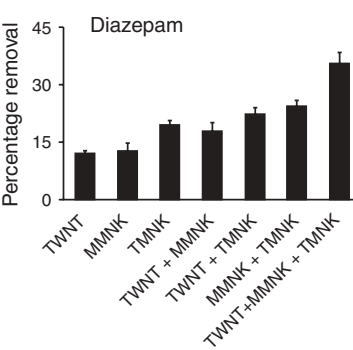

Figure 3 | Cytokines and growth factors produced by the human liver nonparenchymal cell lines. (a) Protein array study demonstrating cytokines and growth factors produced by human liver nonparenchymal cell lines. (b) Endothelial cell line TMNK-1. (c) Cholangiocyte cell line MMNK-1. (d) Stellate cell line TWNT-1. The values are shown as relative intensity of expression compared with an internal control. All cell lines produced IL-6, GR0, IL-8, bFGF, FGF4, FGF9, VEGF, ICAM-1 and TNF alpha, each of which plays a role in liver regeneration. $(\mathbf{e}-\mathbf{g})$ Differentiation and functional assessment of ES cell-derived hepatocytes cultured with different combinations of liver nonparenchymal cell lines. For these studies, the total number of liver nonparenchymal cells used was kept constant for each experiment. Co-culture of mouse ES cells with each cell line (stellate cell line (TWNT-1), endothelial cell line (TMNK-1) and cholangiocyte cell line (MMNK-1)) showed that co-culture with TMNK-1 cells was superior, in terms of generating improvement of cell function, to co-culture with the other two cell lines. Co-culture of ES cells with all three cell lines maximized differentiation toward functional hepatocytes. Data represent function by sorted ALB-GFP-positive cells.

a marker for undifferentiated ES cells, HNF4 and AFP (Fig. 2) should be expressed in EB cells, indicating early development of primitive endoderm in 2-d-old EBs. These results should be consistent with RT-PCR data from D0 cells.

RT-PCR characteristics of differentiating mouse ES-derived hepatocytes (Fig. 2k) from day 0 to day 7 of differentiation should reveal transient expression of the primitive streak gene brachyury, indicating induction of mesendoderm, and induction of markers of definitive endoderm (GSC, Cdh3, FOXA1 and Sox17). Additionally, expression of GATA-4 and HNF-6, common hepatic/pancreatic transcription factors, is induced and maintained until the end of the differentiation protocol (day 14). This suggests that, in addition to differentiation of hepatic lineage cells, the current protocol might also generate other progenitor cell types.

In order to verify induction of definitive endoderm by treatment with Activin A and FGF-2 for subsequent hepatic differentiation (Fig. 2l), we cultured cells for $3 \mathrm{~d}$ with Activin-A only, FGF-2 only, or with neither Activin-A nor FGF-2. Cells were then cultured as outlined above, with dHGF, DMSO and DEX. RT-PCR gene expression on days 7 and 14 of liver-specific ES cell differentiation should show that Activin-A is essential for initiation of hepatic differentiation.

\section{Protein analysis of the culture medium from human liver nonparenchymal cell lines}

See Figure $\mathbf{3}$ for sample results. Cytokines and growth factors produced by the human liver nonparenchymal cell lines should be analyzed to verify that the conditioned medium contains factors needed for hepatic maturation. A protein array study should be performed for this purpose (Fig. 3a-d), and the endothelial cell line (TMNK-1), cholangiocyte cell line (MMNK-1) and stellate 

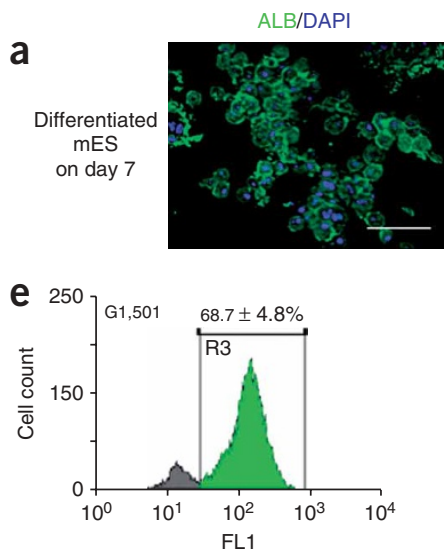

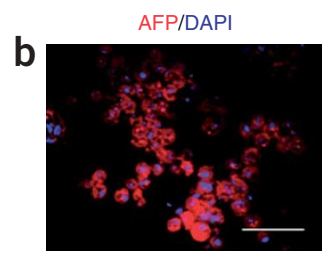

f

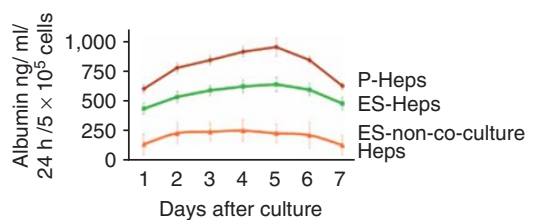

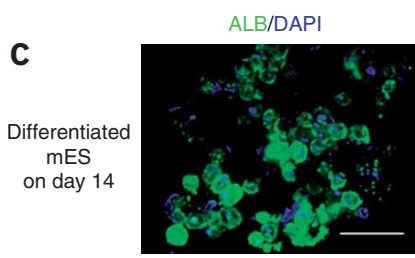

g

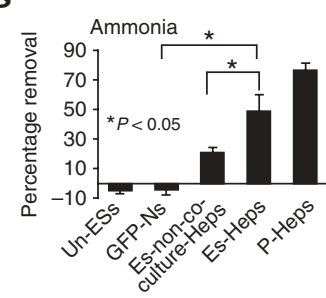

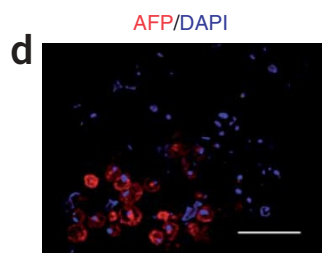

h

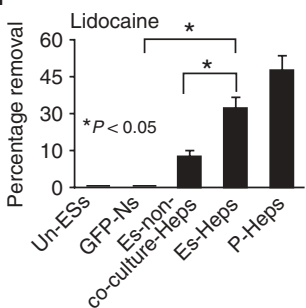

Figure 4 | Immunohistochemical examination of ALB and AFP expression in differentiating EBs. (a-d)

Immunohistochemical analysis of mouse ES ( $\mathrm{mES}$ ) cells at day 7 of the differentiation protocol showed ALB and AFP coexpressed in approximately $48.4 \pm 2.4 \%$ of differentiating cells. By day 14 of hepatic differentiation, $73.0 \pm 6.9 \%$ of the cells expressed ALB, but only $21.3 \pm 3.7 \%$ coexpressed AFP. (e) This data correlated with the data obtained by cell sorting MoFlo after the differentiated cells were transduced with a plasmid containing GFP CDNA under the control of the albumin promoter (pALB-GFP), where by day 14 of hepatic differentiation $68.7 \pm 4.8 \%$ of the cells were GFP-positive. (f) Albumin secretion by ES cell-derived hepatocytes (ES-Heps) sorted for GFP expression from the albumin promoter and mouse primary hepatocytes (P-Heps) $(n=5)$; ES-non-co-culture-Heps: ES cells differentiated without co-culture with liver non-parenchymal cells. (g) Ammonium sulfate $(0.56 \mathrm{mM})$; Un-ESs: undifferentiated mouse ES cells; GFP-Ns: GFP-negative

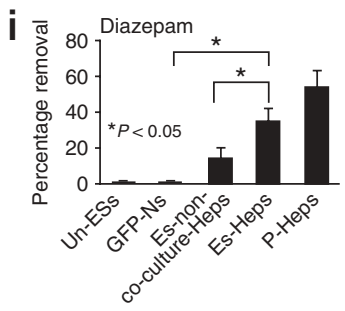
ES-derived cells, (h) lidocaine $(100 \mathrm{mg} / \mathrm{ml})$ or (i) diazepam $(100 \mu \mathrm{g} / \mathrm{ml})$ were loaded into culture wells containing ES cell-derived hepatocytes $\left(5 \times 10^{5}\right.$ cells) sorted for GFP expression from the albumin promoter. The amount remaining was measured $24 \mathrm{~h}$ later and the amount metabolized was calculated per mg cellular protein.

cell line (TWNT-1) should be analyzed. The human nonparenchymal cell lines should produce IL-6, GR0, IL-8, bFGF, FGF4, FGF9, VEGF, ICAM-1 and TNF-alpha, each of which plays a role in liver regeneration. Differentiation and functional assessment of mouse ES-derived hepatocytes cultured with different combinations of liver nonparenchymal cell lines should be assessed in order to find the best conditions for each mouse ES cell line. Co-culture of mouse ES cells with each cell line should show that co-culture with TMNK-1 cells is superior, in terms of improved cell function (Fig. $\mathbf{3 e - g}$ ), to co-culture with the other two cell lines. Co-culture of ES cells with all three cell lines maximizes differentiation toward functional hepatocytes.

\section{Immunofluorescence analysis of albumin/AFP and hepatic function analysis of ES-derived cells}

See Figure $\mathbf{4}$ for sample results. Immunohistochemical analysis of mouse ES cells at day 7 of the differentiation protocol should show that albumin (ALB) and AFP are coexpressed in

Figure 5 | Expression of pancreatic exocrine factors and hormones by ES-cell derived hepatocytes. ES-cell derived hepatocytes did not express gastrin $(\mathbf{a}, \mathbf{d}, \mathbf{g}, \mathbf{j}, \mathbf{m})$, which is normally expressed by mucosal cells in the gastric antrum, or secretin $(\mathbf{b}, \mathbf{e}, \mathbf{h}, \mathbf{k}, \mathbf{n})$, which is normally expressed by $\mathbf{S}$ cells in the proximal small intestine, as determined by immunofluorescence. Similarly, pancreatic amylase $(\mathbf{c}, \mathbf{f}, \mathbf{i}, \mathbf{l}, \mathbf{0})$ was not detected in ES-cell derived hepatocytes (f). Scale bar: $50 \mathrm{~m}$. (p) The production of serine proteases, cysteine proteases, metalloproteases and aspartic proteases was not detected in 24-h cultures of either ES cell-derived hepatocytes or primary hepatocytes (P-Heps). Control 1: $0.15 \mu \mathrm{g} / \mathrm{ml}$ trypsin. Control 2: $0.5 \mu \mathrm{g} / \mathrm{ml}$ trypsin. Control 3: $2.5 \mu \mathrm{g} / \mathrm{ml}$ trypsin. Control 4: $5 \mu \mathrm{g} / \mathrm{ml}$ trypsin. Control 5: $10 \mu \mathrm{g} / \mathrm{ml}$ trypsin.
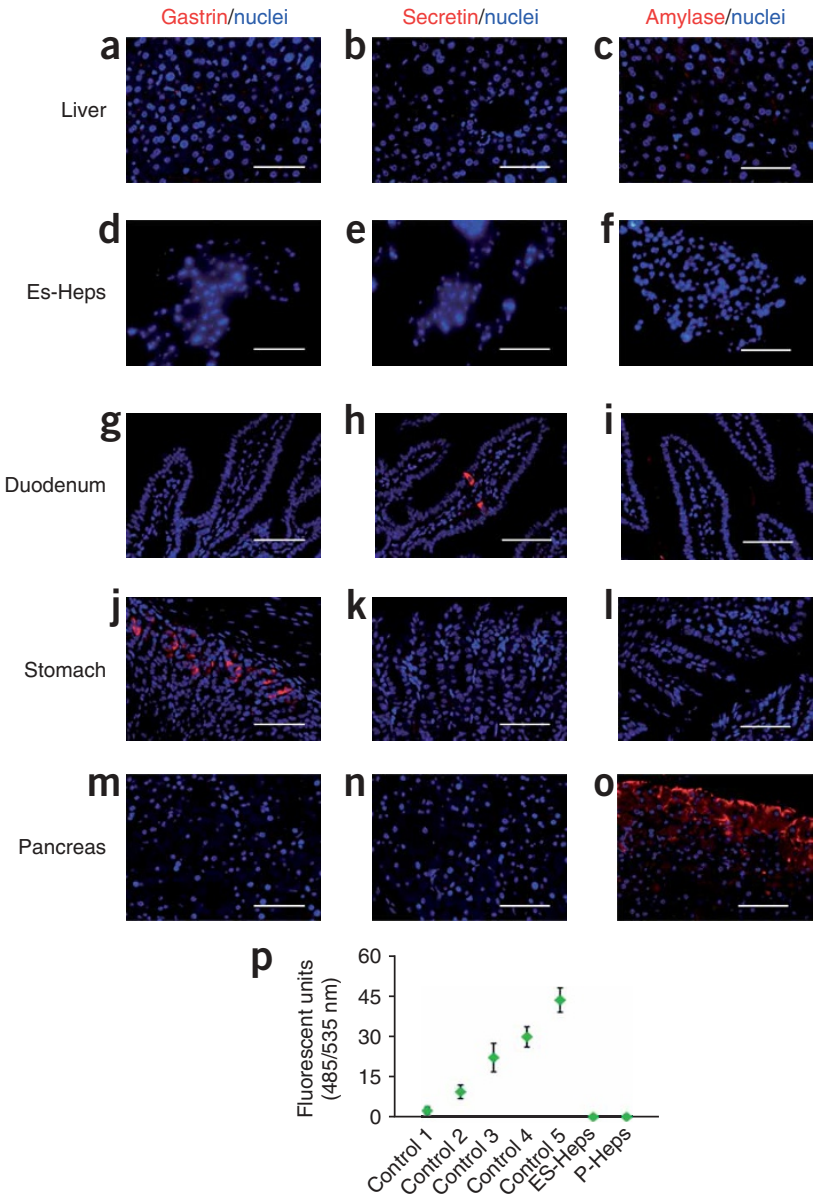
approximately $48.4 \pm 2.4 \%$ of differentiating cells. By day 14 of hepatic differentiation, $73.0 \pm 6.9 \%$ of the cells should express ALB, but only approximately $21.3 \pm 3.7 \%$ should coexpress AFP (Fig. $4 \mathrm{a}-\mathbf{d}$ ). These results should correlate with the data obtained by cell sorting after differentiated cells are transduced with a plasmid containing GFP CDNA under the control of the albumin promoter (pALB-GFP); by day 14 of hepatic differentiation, approximately $68.7 \pm 4.8 \%$ of cells should be GFP positive (Fig. 4e). To show the functional status of the differentiated cells, it should be possible to detect albumin secretion and metabolism of ammonia sulfate, lidocaine and diazepam by mouse ES cell-derived hepatocytes and by mouse primary hepatocytes (Fig. 4f-i). Mouse ES-derived hepatocytes co-cultured with human liver nonparenchymal cell lines showed higher metabolic capacity compared with mouse ES-derived hepatocytes differentiated without co-culture (see ref. 17 and Fig. 3).

\section{Immunofluorescence analysis for pancreatic exocrine factors and hormones by ES-derived cells}

See Figure 5a-d for sample results. Expression of pancreatic exocrine factors and hormones by mouse ES-derived hepatocytes should be performed to characterize the specificity of the differentiation process. Mouse ES-derived hepatocytes should not express gastrin, which is normally expressed by mucosal cells in the gastric antrum, or secretin, which is normally expressed by $\mathrm{S}$ cells in the proximal small intestine. Similarly, pancreatic amylase should not be detected in mouse ES-derived hepatocytes, as determined by immunofluorescence. In addition, the production of serine proteases, cysteine proteases, metalloproteases or aspartic proteases should not be detected in 24-h cultures of either mouse ES-derived hepatocytes or primary hepatocytes (Fig. 5).

\section{Outlook}

Our differentiation system represents a basic protocol to coax mouse ES cells into hepatic lineage cells, and it is amenable to additional strategies for improving in vitro differentiation. This protocol provides a road map for differentiating human ES cells into hepatocytes. The present experimental setup may have applications in addition to those described here. For example, this protocol could be modified to explore induction of different hepatic lineage cells.

Note: Supplementary information is available on the Nature Protocols website.

ACKNOWLEDGMENTS This research was supported in part by a Grant-in-Aid for Scientific Research (B) of the Japan Society for the Promotion of Science to N.K. and NIH grant DK48794 to I.J.F.

COMPETING INTERESTS STATEMENT The authors declare no competing financial interests.

Published online at http://www.natureprotocols.com

Reprints and permissions information is available online at http://npg.nature.com/ reprintsandpermissions

1. Yamamoto, H. et al. Differentiation of embryonic stem cells into hepatocytes: biological functions and therapeutic application. Hepatology 37, 983-993 (2003).

2. Teratani, T. et al. Direct hepatic fate specification from mouse embryonic stem cells. Hepatology 41, 836-846 (2005).

3. Yamada, T. et al. In vitro differentiation of embryonic stem cells into hepatocytelike cells identified by cellular uptake of indocyanine green. Stem Cells 20, 146-154 (2002).

4. Chinzei, R. et al. Embryoid-body cells derived from a mouse embryonic stem cell line show differentiation into functional hepatocytes. Hepatology 36, 22-29 (2002).

5. Kumashiro, Y. et al. Enrichment of hepatocytes differentiated from mouse embryonic stem cells as a transplantable source. Transplantation 79, 550-557 (2005).

6. Lavon, N., Yanuka, 0. \& Benvenisty, N. Differentiation and isolation of hepaticlike cells from human embryonic stem cells. Differentiation 72, 230-238 (2004).
7. Rambhatla, L., Chiu, C.P., Kundu, P., Peng, Y. \& Carpenter, M.K. Generation of hepatocyte-like cells from human embryonic stem cells. Cell Transplant. 12, 1-11 (2003).

8. Kubo, A. et al. Development of definitive endoderm from embryonic stem cells in culture. Development 131, 1651-1662 (2004).

9. D'Amour, K.A. et al. Efficient differentiation of human embryonic stem cells to definitive endoderm. Nat. Biotechnol. 23, 1534-1541 (2005).

10. Yasunaga, M. et al. Induction and monitoring of definitive and visceral endoderm differentiation of mouse ES cells. Nat. Biotechnol. 23, 1542-1550 (2005).

11. Jang, Y.Y., Collector, M.I., Baylin, S.B., Diehl, A.M. \& Sharkis, S.J. Hematopoietic stem cells convert into liver cells within days without fusion. Nat. Cell Biol. 6, 532-539 (2004).

12. Poll, D.V. et al. Elevated hepatocyte-specific functions in fetal rat hepatocytes co-cultured with adult rat hepatocytes. Tissue Eng. 12, 2965-2973 (2006).

13. Kang, Y.H., Berthiaume, F., Nath, B.D. \& Yarmush, M.L. Growth factors and nonparenchymal cell conditioned media induce mitogenic responses in stable long-term adult rat hepatocyte cultures. Exp. Cell Res. 293, 239-247 (2004).

14. Matsumura, T. et al. Establishment of an immortalized human-liver endothelial cell line with SV40T and hTERT. Transplantation 77, 1357-1365 (2004).

15. Watanabe, T. et al. Establishment of immortalized human hepatic stellate scavenger cells to develop bioartificial livers. Transplantation 75, 1873-1880 (2003).

16. Maruyama, M. et al. Establishment of a highly differentiated immortalized human cholangiocyte cell line with SV40T and hTERT. Transplantation 77, 446-451 (2004).

17. Soto-Gutierrez, A. et al. Reversal of mouse hepatic failure using an implanted liver-assist device containing ES cell-derived hepatocytes. Nat. Biotechnol. 24, 1412-1419 (2006). 